\title{
Exploring classroom interaction with dynamic social network analysis
}

Christian Bokhove

Southampton Education School, University of Southampton, Southampton, United Kingdom

Highfield Campus, Building 32 Room 2035, Southampton, SO17 1BJ, C.Bokhove@soton.ac.uk 


\section{Exploring classroom interaction with dynamic social network analysis}

This article reports on an exploratory project in which technology and dynamic social network analysis (SNA) are used for modelling classroom interaction. SNA focuses on the links between social actors, draws on graphic imagery to reveal and display the patterning of those links, and develops mathematical and computational models to describe and explain those patterns (Freeman 2004). Dynamic SNA extends SNA and builds on previous research on network change (e.g. Moody, McFarland, and Bender-deMoll 2005). Utilizing data from six videos from the TIMSS video study (Hiebert et al. 2003) and a convenience sample of five observations of one teacher in a secondary school in the south of England, a methodology for dynamic SNA was applied to classroom interaction data. The results are in two ways relevant for this journal. Firstly, they show that recent developments in social network analysis might be usefully applied to furthering knowledge about the dynamics of classroom interaction. Secondly, the results provide an example of the use of tablet technology for fieldwork data collection.

Keywords: classroom interaction, technology, dynamic social network analysis

\section{Introduction}

This article reports on an exploratory project that uses technology and social network analysis (SNA) for modelling classroom interaction. The article argues that SNA might be a useful candidate for exploring classroom interactions. In addition to the methodological usefulness of SNA, it also proposes that technology can play an important role in both capturing classroom interaction and analysing the resulting data. The article will first describe the relevance of studying classroom interaction and establish some methodological history in studying it. After the principles and appropriateness of SNA is described with some fictional data, the article discusses technological and methodological aspects of capturing classroom interaction with SNA. Finally, three analyses using video data and data from an observation app are described, 
finishing with conclusions and discussion of the challenges and discussion of the proposed methodology.

\section{What do we mean by classroom interaction?}

This paper starts off to describe the important role of classroom interaction and teacher effects. (Muijs and Reynolds 2010; Scheerens and Bosker 1997; Teddlie and Reynolds 2000). Some of the variables included in the dynamic model of educational effectiveness indicate that the interactions taking place in the classroom between students and teacher play an important role (Creemers and Kyriakides 2008). This importance is further emphasized by Hattie’s (2009) list of effective teaching practices. Interactions between actors in the classroom are important, for example in providing formative evaluation, giving feedback and in teacher-student relationships. One component of interaction is classroom dialogue, something which is apparent in Howe and Abedin’s (2013) inclusive approach to the term classroom dialogue. In their definition, classroom dialogue resembles the notion of interaction presented by Scott and colleagues (Scott, Mortimer, and Aguiar 2006; Scott and Ametller 2007) and is not restricted to talk. Analysing dialogue could strongly contribute to understanding important classroom interactions. From that starting point this article sees dialogue as an ingredient of interaction but will use the terms interchangeably. Another discussion point regarding ‘interaction’ concerns whether a reply is necessary to call an utterance an interaction. Howe and Abedin (2013) conceptualized dialogue as "all verbal exchanges where one individual addresses another individual or individuals and at least one addressed individual replies.” (2013, 326). According to this definition something like 'teacher praise’ would not be considered part of classroom dialogue, as there certainly will be occasions where praise is not followed by 'at least one individual reply’. As this article wants to take an inclusive approach to recording classroom 
interactions, it takes an interaction to be "every verbal uttering from one actor to another”. Apart from the definition of classroom interaction, historically research into classroom dialogue has resided on the boundary of quantitative and qualitative approaches.

\section{Quantitative and qualitative approaches in analysing classroom interaction}

Howe and Abedin (2013) describe how research on classroom dialogue in the last four decades concerned both teacher-student and student-student interactions, and that from 1972 to 1992 there was an emphasis on analytical quantitative approaches, and mainly qualitative from 1992 to 2011. The quantitative approach mainly involved field coding, whereby observation is contained within some predetermined system of categories, which was heavily criticized in the late 80s (see Edwards and Westgate 1994, for a summary). The main objections against field coding were that it isolated utterances from their broader context and that it did not take into account the temporal sequence of utterances. Howe and Abedin (2013) describe the total dismissal of these methods as 'arguably ill advised', as coding schemes can be used reliably and repeatedly by different researchers, 'permitting comparison across a wide range of contexts' (2013, 222). In the conclusions of the review they argue that models of optimal practice should be studied, i.e. whether some models of dialogic organization are more beneficial than others. Furthermore, as there are few 'target-based' studies, often aimed at small groups rather than whole classes, this research has to be prioritized. The authors also argue that target-based assessment of dialogic practices through qualitative means is very difficult. In principle, it is suggested, the problems can be overcome using quantitative means and statistical techniques, while keeping in mind its limitations as well. It is acknowledged that there is widespread unease about quantitative analysis in relation to dialogue. Rather than solve this dilemma $a$ priori they propose to 'take a risk' and see whether 
quantification is a useful proxy for classroom dialogue or whether the simplification is crippling. The author of this article suggests that a 'network approach' might be a fruitful way to combine quantitative and qualitative approaches of classroom interaction. In essence, this article then tries to address the challenge posed in Howe and Abedin's (2013) article by proposing a methodology that:

1. Allows for comparisons across a wide range of contexts (See TIMSS video study section for the approach that is envisaged).

2. Includes the temporal aspect: sequences of interactions are taken into account.

3. Can be used in a whole class situation.

4. Can include relevant variables like gender and attainment as well, so they can be included in statistical models.

5. Does not preclude the inclusion of qualitative data.

6. Adds a visual aspect to classroom interaction.

This method involves social network analysis (SNA).

\section{Using SNA to describe classroom interaction}

Social network analysis (SNA) looks at social relationships in terms of network theory, consisting of nodes, representing actors within the network, and ties (or edges) which represent relationships between the actors. Originally the concept of 'social networks' has been studied since the early 20th century to explore relationships between members of social systems. In more recent years, social network analysis has found applications in various academic disciplines, as well as practical applications such as countering money laundering and terrorism. It is outside the scope of this article to give an overview of the whole field; for this there are basic introductory texts on SNA (e.g. amongst others, Prell 2011; Scott 2013; McCulloh, Armstrong, and Johnson 2013). Freeman (2004) reviewed the development of SNA from its earliest beginnings until the 
late 1990s. He characterizes SNA as involving four things (i) the intuition that links among social actors are important, (ii) it is based on the collection and analysis of data that record social relations that link actors, (iii) it draws heavily on graphic imagery to reveal and display the patterning of those links, and (iv) it develops mathematical and computational models to describe and explain those patterns. Actors are usually represented as points (nodes), and relations among actors are represented by lines (edges), with relational direction indicated by arrows. SNA maps and measures relationships and flows between people, groups, organizations, computers, and other connected entities, and allows for both a visual and mathematical analysis of human relationships. SNA has been used in education before, with a sizable amount of research on networks within and between schools. For instance, a network approach has been applied to research on managing teacher professional communities (e.g. Penuel, Krause, and Frank 2009), the influence of policy on teachers’ social networks (e.g. Coburn and Russel 2008), school redesign networks (e.g. Daly and Finnegan 2010) and school leadership (e.g. Pitts and Spillane 2009). Other SNA research has been aimed at primary and secondary classrooms, and if it was it mainly focussed on peer influences (who likes or dislikes who) and bullying (e.g. Sentse, Kiuru, Veenstra, and Salmivalli 2014). By no means is this meant as exhaustive review of SNA applications in education. It does, however, frame the current article as one in that tradition of applying SNA to another focus of study: classroom interactions.

Li, Shouhui and Xinying (2010) propose that from the network perspective, teacherstudent interactions in the classroom can be seen as a verbal communication network, whereby verbal communications are perceived as connections (edges or ties) between individual student and the teacher. Actors like students and teacher are seen as nodes or vertices in the network. The structure of the in-class interactions can be studied through 
analysing the basic attributes of the network. Li, Shouhui, and Xinying (2010) also suggest that SNA can be applied to analysing the classroom environment with the data acquired from classroom observation systems. SNA could provide several metrics that might be applicable to classroom interactions. The application of SNA to classroom interaction is demonstrated by the fictional network in figure 1 of one teacher T01, and seven students S01 to S07, six nodes in total. The nodes can have attributes, for example gender, which is indicated by a colour (blue=female, pink=male).

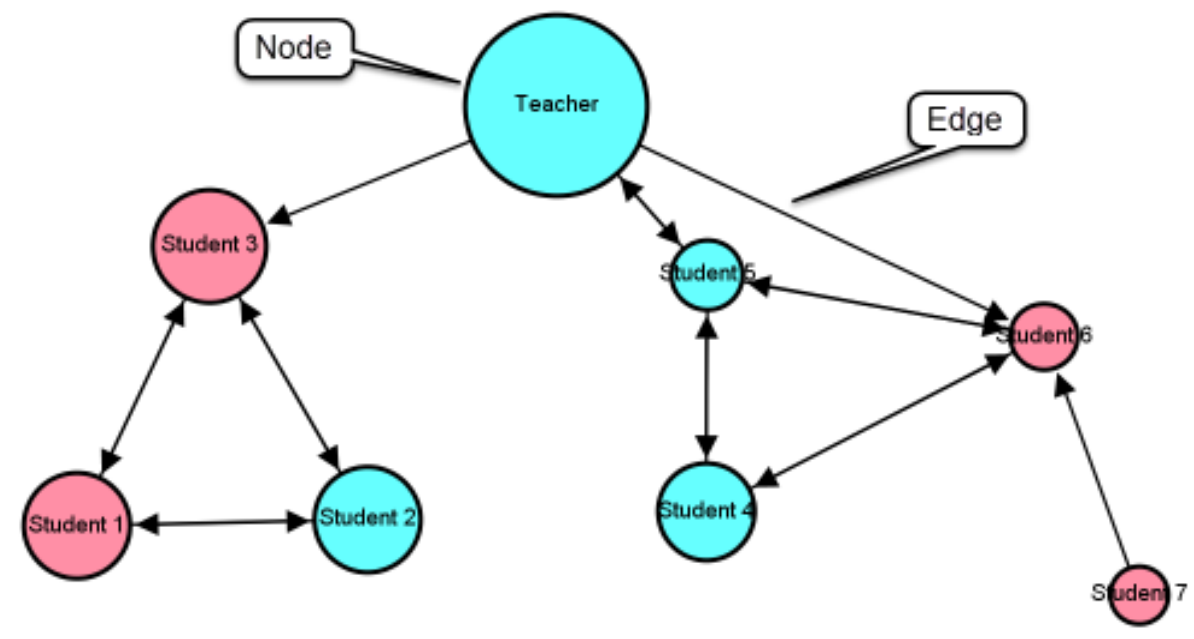

Figure 1. Fictional data for one teacher and seven students and interactions.

In this fictional example the teacher has interacted with S03, S05 and S06, visualized by the edges between nodes. S05 has asked the teacher a question so there is an arrow from S05 to the teacher as well. The students S01 to S03 and S04 to S07 worked and interacted in distinct groups, while S07 only interacted with S06. It is appropriate to assume that interactions go from one node to another, a directed edge, rather than an undirected one. The figure gives a snapshot of the interactions between the different actors. Visually, the social network already conveys information, as it, for example, shows there are two distinct groups of students, and one solitary student. Of course, this network is subject to constant change. Rather than giving qualitative descriptions of a snapshot network, measures can be calculated as well. An example might be the number 
of edges. If an edge represents an interaction then the number of edges represents the amount of interaction taking place in a classroom. The degree of an actor (a node) then is the number of edges that run from or into a certain node. Depending on directionality one can distinguish an in-degree as the edges that go into a node and out-degree as the edges go from a node. The degree can tell us something about the involvement of an actor in classroom interaction. For example, a teacher that tends to use a lot of classroom instruction, might have a high out-degree: interaction mainly runs from the teacher to the students. In figure 1 T01 has an out-degree of 3 and an in-degree of 1 . S07 has an in-degree of 0 which indicates no one interacted with that student. Another metric that might tell us something about the amount of classroom interaction taking place is the average degree, the mean of all the degrees in a network (in figure 1 this is 2.125). A higher degree is not always better; more often it is more interesting to see which actors are the most central in a network and how essential these actors are for the network, so-called nodal centrality measures. Drawing from McCulloh et al. (2013) degree centrality might be seen as the assumption that the node with the most edges/ties holds a special place of influence within a network. Betweenness centrality measures how often a node appears on shortest paths between nodes in the network (in figure 1 S05 has the highest value). It can indicate how 'needed' a node is in the communication within a classroom i.e. a broker function for a certain student. Finally, Eigenvector Centrality is a measure of node importance in a network based on another node's connections (in figure 1 S06 has the highest value) i.e. 'connectedness to other wellconnected people’. Nodes with a high eigenvector centrality are considered to be influential nodes in a network, e.g. influential students in the classroom. It is also possible to study 'sub-groups' by looking at modularity which measures how well a network decomposes into modular communities, or the clustering coefficient which is a 
measure of the degree to which nodes in a graph tend to cluster together. Both can convey a sense of group formation. Essentially, the claim is that if we perceive classroom interaction as a social network, metrics from SNA might be useful for describing classroom interaction patterns, and exploring groups and positions within classrooms. Further examples of this will be given later on in the article. In addition, a range of tools becomes available to visualize this classroom interaction. One thing still missing, however, is the dynamic aspect of classroom interaction: classroom interaction happens over time, during the course of a lesson. What might SNA offer when it comes to these dynamics?

\section{Adding a dynamic aspect to SNA}

Most research that utilizes social network analysis studies relatively static notions of networks (Bender-deMoll and McFarland 2006). Even when studies looked at changes over time (see Doreian and Stokman 1997 for a review), data was collected periodically and the intervals between data collection points was quite long. In the beginning of this millennium, several developments led to an increasing interest in the temporal evolution of networks. Bender-deMoll and McFarland (2006) describe these as: innovations in data collection (e.g. Choudhury and Pentland 2004), the application of network methodologies to new fields (e.g. Barabasi 2002; Dunne, Williams, and Martinez 2002), and the use of simulations as data sources (e.g. Banks and Carley 1996; Jin, Girvan, and Newman 2001). These developments also gave rise to advances in theory development (e.g. Moody, McFarland, and Bender-deMoll 2005), and refinement of statistical and modelling techniques (e.g. Wasserman and Pattison 1996; Snijders 1996; 2001). All these developments indicate that the field of dynamic SNA has developed considerably, allowing the study of the relationship between interaction and network social structure. It also presents a new set of challenges for conceptualizing, visualizing, and 
communicating information about social networks. For classroom interaction a prime example is given by the Moody, McFarland and Bender-deMoll (2005). This study

utilizes data from McFarland's repeated observations of social interactions in over 150 high school classrooms during the 1996-97 school year. The study presents dynamic network representations of social interaction from several classes. The study suggests that a study of dynamic networks conveys information about features like the level of control by the teacher and the level at which teachers are involved in engaging their students. These patterns of classroom interactions are exemplified by animations that show network development. Now that dynamic SNA has been presented as methodology for analysing classroom interaction, it is necessary to describe how classroom interaction data can be captured.

\section{Observation methods to capture classroom interaction}

The present study attempts to utilize two often used methods for observation, indirect and direct methods (Cohen, Manion, and Morrison 2011). The first is indirect in that it involves recorded classrooms and analysis afterwards, for example with video and transcripts. The second approach involves direct observation through 'real time' data collection in the classroom, using a mobile app. In both cases one has to deal with recording actual behaviours allowing for fine-grained exploration of behaviours (Muijs and Reynolds 2010). There are several reasons to include these two distinct phases. Firstly, the phase with existing video data serves as a means of piloting the computer program(s) for visualising networks. Secondly, as it concerns a credible set of video from actual classroom practice, the methodology might potentially allow already recorded (and released with transcript available) videos to be re-analysed. 


\section{The use of video for indirect observation: the TIMSS video study}

Although this article looks at a new methodology with technology and Social Network Analysis (SNA) it is not the only way to analyse classroom interaction nor is it the first attempt. The first phase of this project has been inspired by the 1999 TIMSS video study. The Third International Mathematics and Science Study (TIMSS) 1999 Video Study is a follow-up and expansion of the TIMSS 1995 Video Study of mathematics teaching (Hiebert et al. 2003). It involved grade eight mathematics and science teaching in seven countries; Australia, the Czech Republic, Hong Kong SAR, the Netherlands, Switzerland, and the United States. At the time it garnered attention through its novel methodology, in which national samples of teachers were videotaped teaching an eighth-grade mathematics lesson in their regular classrooms, and a focus on the differences and similarities in teaching among the participating countries. The TIMSS study aimed to develop objective, observational measures of classroom instruction to serve as appropriate quantitative indicators of teaching practices in each country, to compare teaching practices among countries and identify similar or different lesson features across countries; and to describe patterns of teaching practices within each country. This ambition is reflected in the report National Patterns of Teaching (Givvin, Hiebert, Jacobs, Hollingsworth, and Gallimore 2005) where the authors tried to infer general patterns of teaching between countries. They proposed that key to making progress on uncovering national or global patterns, if they exist, is to examine actual classroom teaching. The authors specifically took into account durations and sequencing of events in 638 lessons from randomly selected and nationally representative classrooms, and combined them in so-called 'lesson signatures'. This way it was felt it was possible to unpick what are universal elements and what are cultural aspects and beliefs. The report suggests that the 'easiest' way to compare countries this way is to use quantification. For example, field coding episodes of public and private interaction 
shows differences and similarities between countries. Across countries length of time of public interaction (whole classroom) varied. Although the fraction public/private interaction was quite similar, there were differences in sequencing and duration. The TIMSS study has also been influential from a methodological point of view, especially with regard to video analysis (Janík and Seidel 2009). Hiebert et al. (2003) mention several affordances of video study that counteract the disadvantages of direct observations. Advantages are:

- video enables the study of complex processes

- video increases inter-rater reliability, decreases training difficulties

- video enables coding from multiple perspectives

- video stores data in a form that allows new analyses at a later time;

- $\quad$ video facilitates integration of qualitative and quantitative information;

- video facilitates the communication of the results.

The TIMSS video study establishes a case for the observation of classroom interaction and use of video analysis. However, the study also reported several pitfalls (Jacobs, Hollingsworth, and Givvin 2007) ranging from technical issues, clear protocols, ethical aspects to filming permissions. The latter also gave rise to, apart from analysing existing videos, a second observation method with observation apps. Anecdotally, schools that were approached for this project were more willing to agree to direct observation with an app than with video.

\section{Technological advances for direct observation: observation apps}

Technological advances have made it easier to model classroom interaction with direct observational methods. The developments are apparent in tools that are available for capturing (classroom) interaction in such a way that they can be easily used for 
subsequent social network analyses. Early candidates in this project were the Work Activity Observer App for the iPad (Hansberger 2012) and the HART app (Baker, Salvi, Van Velsen, and Whiting 2013). However, because of the more extensive features with regard to taking notes, as well as better data export functions, this project was conducted with the Lesson Note from the Lesson Study Alliance (2014). Their Lesson Note app was deemed particularly useful because the way interactions are recorded closely resembles a network approach (Bokhove 2015). For example, the app allows for the creation of seating charts with students, teachers, desks and inventory. Furthermore, the app allows interactions to be indicated by moving one’s finger (swiping) from one node to another (see figure 2a). Automatically, time stamps are added to these swipe actions, allowing the observer to record interactions over time.

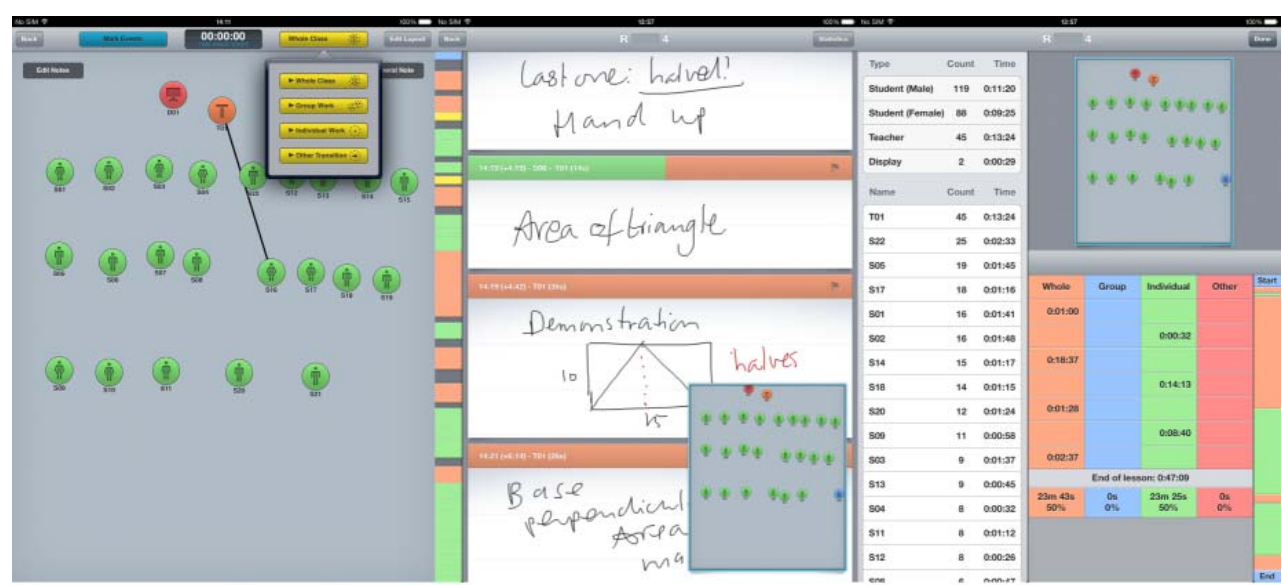

(a) (b) (c)

Figure 2. Overview of some of Lesson Note's features: (a) input interactions through swiping, (b) adding notes to interactions, and (c) basic statistics of a captured lesson.

Hand-written and drawn notes and images can be associated with the interactions (see figure 2b). To designate different 'phases' of the lesson transitions can be indicated, like 'whole class' or 'individual work' (figure 2a). After having finished observing a lesson some basic statistics for the lesson can be presented (figure 2c). Data can be exported in such a way that it can be processed into a format that, with some modifications, can be used for SNA. The use of the app is further demonstrated in the results (analysis C). 


\section{Methodological advances: analysing networks}

Methodological advances have also made it easier to model classroom interaction, as technology has been conducive for several methodological improvements when studying both sets of static networks and dynamic social networks. Advances in modelling techniques have resulted in numerous software packages for visualizing social networks and providing basic metrics. It is beyond the scope of this article to describe all the packages available (for example UCI-net, Pajek). In this study the main program used was Gephi (Gephi Consortium 2014), an “interactive visualization and exploration platform for all kinds of networks and complex systems, dynamic and hierarchical graphs”. Figure 3 gives an impression of Gephi’s user interface.

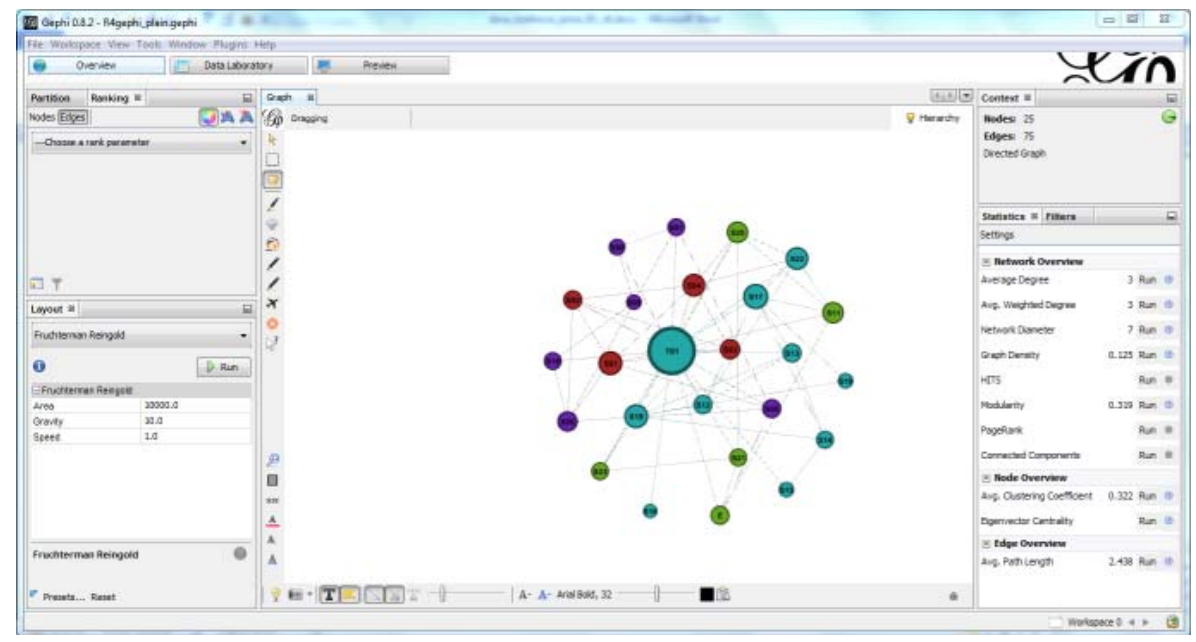

Figure 3. An impression of the Gephi user interface with classroom interaction data.

There also have been numerous advances in statistical models for social networks and network dynamics. Given the challenge described in the introduction it might be fruitful to use these models to describe patterns of classroom interaction. One category of model concerns exponential-family random graph models (ERGMs) or p-star models (Wasserman and Pattison 1996; Robins, Pattison, Kalish, and Lusher 2007). These models allow for estimates for the parameters of a specified model for a given data set, simulate additional networks, test models for goodness-of-fit, and perform model comparisons. An extension of these models is represented by separable temporal 
ERGMs (STERGMs), useful for modelling dynamic networks in discrete time (Krivitsky and Handcock 2010). Another approach for modelling the dynamics of a network (or of networks and behaviour) is an actor-oriented model (Snijders 2001). Software packages for all these models are available in R, a free and open source software programming language and software environment for statistical computing (e.g. statnet by Handcock, Hunter, Butts, Goodreau, and Morris 2008; RSiena by Ripley, Boitmanis, and Snijders 2013). All these methods have in common that they can model changes in networks over time, and therefore can all provide useful avenues to make a case for the potential of dynamic SNA to analyse classroom interaction. In the present study we will only use a subset of these methods, but contend that potentially all can be used after establishing a 'proof of concept'. This will be done by first utilizing existing classroom video data from the TIMSS study, and then by using new data from direct classroom observations with the Lesson Note app. By doing both, it is expected that the methodology can be useful for both old and new data. Furthermore it provides insight in the way the methodology was developed.

\section{Methodology}

\section{Source data}

The project uses two sources of data. The first, dataset I, consists of classroom videos and transcripts from the TIMSS 1999 Video Study. The chosen videos, three from the USA and three from Hong Kong SAR, are available on the http://timssvideo.com website. As videos/transcripts of US1 and HK1 are used extensively in the examples in the results section it seems to make sense to describe the videoed lessons. US1 is an USA eighth grade mathematics lesson which focuses on graphing linear equations. It is a review lesson that follows a unit of work on this topic. The lesson is 44 minutes in 
duration. There are 36 students enrolled in the class. For the most part, the teacher circulates from group to group, assisting students throughout this period of 'private interaction'. In this lesson, students work in groups during all of the private interaction periods. HK1 is a Hong Kong SAR eighth grade mathematics lesson with a focus on square numbers and square roots. It is the second in a sequence of lessons working towards Pythagoras' theorem and its applications. The lesson is taught in English and lasts for 34 minutes. There are 40 students in the class. In the first part of the lesson the focus is on public whole classroom interaction. After teaching the concept at hand students are asked whether they had any questions. A lack of response is finally followed by a request from the teacher to do some exercises.

The second source, dataset II, consists of five exported Lesson Note app observation files in csv format from five mathematics lessons given by one teacher at an independent school in the south of England. The five observed lessons are from year 7, 9, 10, 12 and 13 respectively, covering topics like Pythagoras and ratios. A further typology of the lessons is provided by presenting the results of the lessons, for example in Table 3 and the supplemental materials. In this study the aim was not so direct comparability, as evidenced by the different year groups used, but the potential for a standardised methodology to represent classroom interaction.

\section{Data analysis and visualization software}

The study used Gephi 0.8.2 beta, R and Rstudio with the packages statnet (Handcock, Hunter, Butts, Goodreau, and Morris 2008) and ndtv (Bender-deMoll 2014). Several visualizations were created as well as metrics, both static and over time. As layout algorithm in Gephi Fruchterman-Reingold was used (Fruchterman and Reingold 1991). 


\section{Data preparation}

To be able to analyse the data it was necessary to convert the data into a network format that would, at a minimum, include information about nodes and edges. In most formats edges are indicated by pairs of sender and receiver nodes. Edges are indicated as directional, meaning that the pair A,B is different from B,A. Both nodes and edges are further supplemented by start and end times denoting the temporal aspect. Nodes and edges do not have any additional attributes, apart from gender and edge duration. Events of duration 0 are removed from dataset II: these are actions by solitary students.

\section{Three data analyses approaches}

Analysis A uses the TIMSS data at the detail level of the existing transcripts. The six transcripts from dataset I were used. In figure 4 the data processing procedure is demonstrated. At the top there is the existing text transcript with timestamp, the sender (T for teacher, SS for the whole class, and SN and S for individual students) and the utterance. As social network data types also need a 'target node', the intended 'receiver' was as good as possible inferred from the transcript. Furthermore, some edges were aggregated; SS was recoded to E for 'entire class' and SN to S for 'student'. The interim result is in the middle of figure 4 . Finally the timestamps were converted to start- and end times. An extra column was calculated by subtracting start time from end time, allowing the duration of the utterance to be used as weight. Every line in the file was perceived as one interaction. The data was then processed in Gephi. 


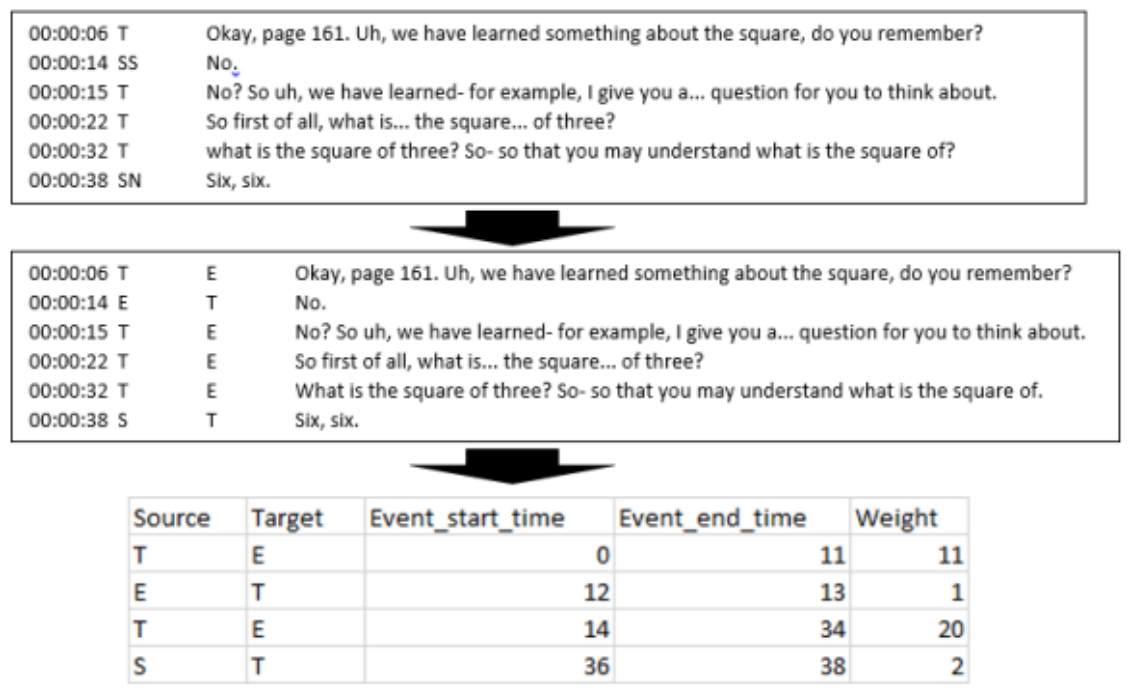

Figure 4. Example of data processing for analysis A.

In analysis B two TIMSS videos from dataset I, US1 and HK1 were re-observed to supplement the existing transcripts with specific student details: what were students and teachers saying at specific time points and who was saying what. Senders and receivers 'S' now became S1, S2, S3, etc. A classroom plan was used to assist the observation. After this the data from this was taken and used in Gephi and statnet (specifically the package networkDynamic) and ndtv packages within Rstudio. In analysis $C$ the five observations from dataset II are taken and processed in a similar way as analysis B with Gephi. The exact protocol for data processing is beyond the scope of this paper; it primarily involved getting the timestamps in an appropriate format for Gephi. In the results section the methodological aspects of our analyses are described and summarized, as well as methodological challenges that were encountered.

\section{Results}

\section{Analysis A}

Visualizations as well as network metrics of videos/transcripts US1 and HK1 are presented in figures 5 and 6, and table 1 respectively. In figures 5 and 6 the curve 
indicates the direction of an edge: read an edge clockwise from a source node to a target node.

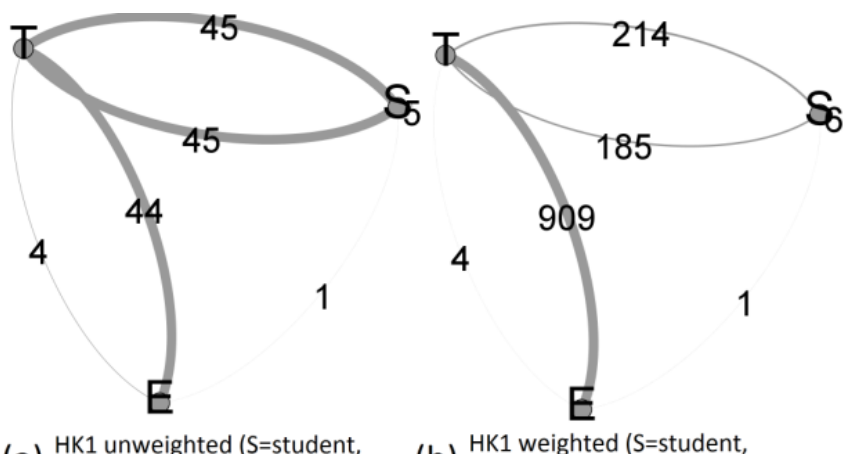

$\begin{array}{ll}\text { (a) }{ }_{\mathrm{T}=\text { teacher, } \mathrm{E}=\text { entire class) }}^{\mathrm{HK} \text { unwed }} & \text { (b) }{ }_{\mathrm{T}=\text { teacher, } \mathrm{E}=\text { =entire class) }}^{\mathrm{HK} 1 \text { weighted (S=student, }}\end{array}$

Figure 5. Visualization for HK1.

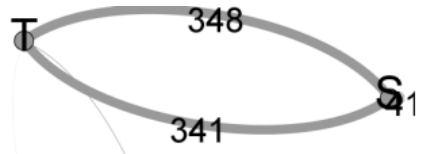

26

(a) $\quad \mathrm{E}$

US1 unweighted (S=student, T=teacher, $\mathrm{E}=$ entire class)

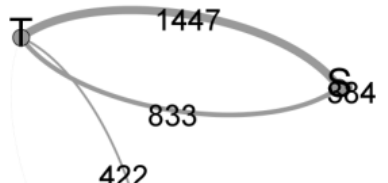

2

E

(b) US1 weighted ( $\mathrm{S}=$ student,

$\mathrm{T}=$ teacher, $\mathrm{E}=$ entire class)

Figure 6. Visualization for US1.

Table 1. Edge information, unweighted and weighted by duration, of US1 and HK1.

\begin{tabular}{|l|l|c|c|c|c|}
\hline & & \multicolumn{2}{|c|}{ HK1 } & \multicolumn{2}{c|}{ US1 } \\
\hline From & To & Unweighted & Weighted & Unweighted & Weighted \\
& & & by duration & & duration \\
\hline T & E & 44 & 909 & 26 & 422 \\
\hline T & S & 45 & 214 & 348 & 1447 \\
\hline E & T & 4 & 4 & 2 & 833 \\
\hline S & T & 45 & 185 & 341 & - \\
\hline S & E & 1 & 1 & - & 384 \\
\hline S & S & 5 & 6 & & \\
\hline
\end{tabular}


Although the data only consists of a limited level of detail, the data shows a much higher weight for Teacher-to-Entire-class (T-E) interactions in the HK1 than US1, 909 versus 422. For Teacher-to-Student interactions this pattern is reversed (214 versus 1447), justifying US1 to be typified by a strong teacher-student interaction. Both correspond with the general descriptions of the lessons. The difference between the unweighted and weighted network seems to indicate that there is difference in the duration of the interactions. However, apart from observational challenges and choices, it is also clear that using a limited number of aggregate nodes does not convey much about the dynamics in a classroom between the actors. To obtain this we need to have more fine-grained data from the TIMSS video study. This could also give an indication of possible cliques and clustering (i.e. whether there are groups of student that interact more or less). Analysis B extends the analysis.

\section{Analysis B}

Visualizations as well as network metrics of US1 and HK1 are presented in table 2.

Table 2. Networks and some basic metrics for HK1 and US1.

\begin{tabular}{|l|c|c|}
\hline & \\
& Figure 7. Visualization for HK1. & Figure 8. Visualization for Us1. \\
\hline Nodes & 44 & \\
\hline Edges & 51 & \\
\hline Average degree & 1.159 & 2.714 \\
\hline
\end{tabular}


These visualizations confirm the findings of analysis A; HK1 contains relatively few interactions (Figure 7) and there is an emphasis on teacher-wholeclass interaction. In US1 there is an emphasis on interaction between teacher and individual student(s) and among students (Figure 8). Overall, the number of interactions is much larger in US1 than in HK1. Taking the duration into account even further inflates the average weighted degree of US1 compared to HK1. Visually, the US1 network immediately conveys a different interaction pattern than HK1. One point to note in this analysis was that re-observing the videos proved to be a considerable challenge when using video data, confirming many of the challenges Hiebert et al. reported (2003):

- The camera angle keeps changing.

- The teacher seems to talk to groups sometimes. How do you capture this? Should groups be perceived as individual entities or not?

- When does a teacher actual talk to the whole class?

- The camera is not faced at the person talking so it's hard to deduce which person is actually talking. This is exacerbated if the utterance is in a foreign language.

- Does one record interactions that constitute of students talking themselves?

- Does one record all off-task interactions between students?

- Is it even possible to record all interactions?

With better video and observation protocols some of these challenges might be addressed. In addition, multiple camera viewpoints are desirable. Some disadvantages could also be mitigated by using direct observation, rather than video. The dynamic aspect in Gephi proved to be more difficult to model than expected, as Gephi aggregates 
edges over time. This means that the edges at different time points are combined into one, losing some data about time. For static snapshots as figure 7 and 8 in table 2 this does not pose a problem as the temporal aspect cannot be included any way, but obviously to track metrics over time this is needed. To visualize the dynamic aspect socalled networkDynamic objects of the two lessons were created with the statnet package in R (Handcock, Hunter, Butts, Goodreau, Krivitsky, Bender-deMoll, and Morris 2014). After that the object was used in the ndtv package's animation, 'timeline' and ‘filmstrip’ functions (Bender-deMoll 2014). The result of the latter is depicted in figure 9.
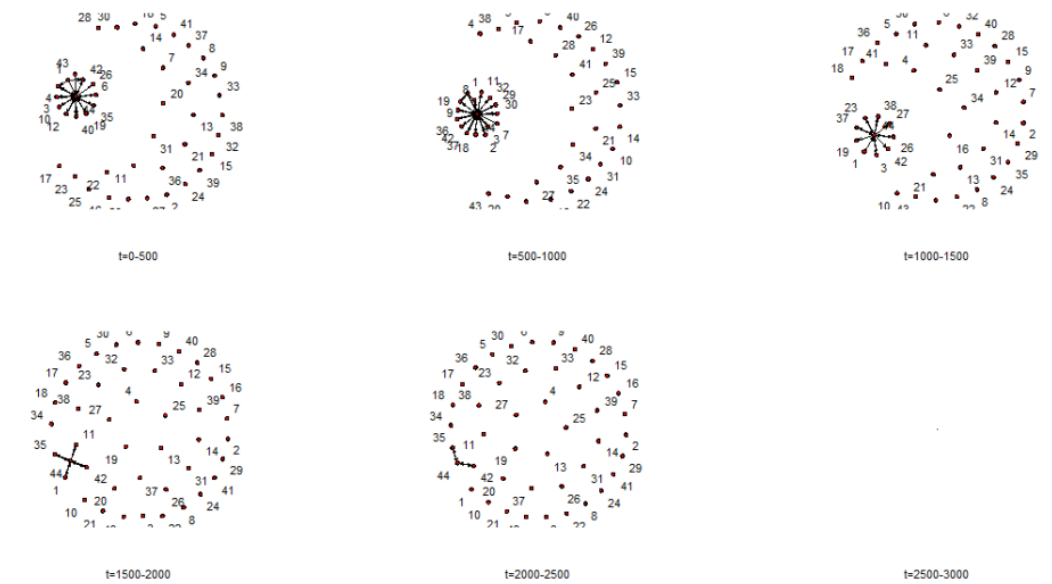

Figure 9a. Filmstrip representing an animation for $\mathrm{HK1}$ created with statnet and ndtv in $\mathrm{R}$.
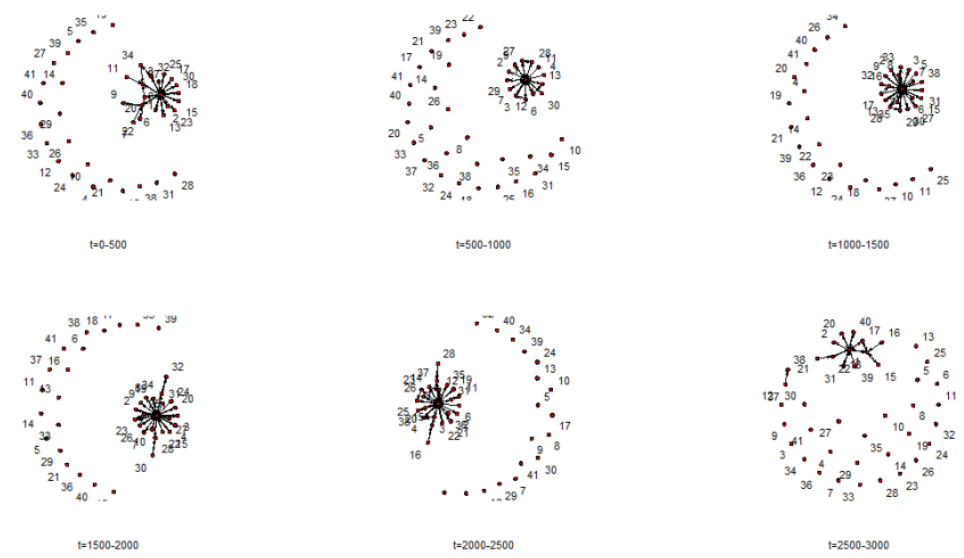

Figure 10b. Filmstrip representing an animation for US1 created with statnet and ndtv in R.

The conclusions of the static network versions are confirmed in this dynamic model; there is more interaction during the lesson, demonstrated by more edges throughout the 
longer US1 lesson. In addition, the number of nodes, the direction of the edges and the edges between teacher and student suggest there is a more complex interaction pattern in US1 compared to HK1. Note that one node still represents ' $E$ ', the entire classroom. One could argue that edges from the teacher to all students would be a more appropriate representation. In further analyses multiple metrics could be calculated over time, as well as statistical models fitted.

\section{Analysis C}

Five mathematics lessons were captured with the Lesson Note app, yielding a total number of 1087 notes. These notes consist of hand-written notes, images and an indication of interactions taking place. At the end of every lesson a 'holistic' summary of all five lessons was written in a journal as well. I wanted to use these to 'check' whether the SNA approach could corroborate my overall impression of the lesson. The exported notes were processed to a social network format and imported in Gephi. As an example two out of five networks are presented in the paper, the three additional lessons are available as supplemental material. The first lesson (R1) is a year 10 maths lesson on proportions. The lesson can be typified as being mostly teacher led, with some whole-classroom interaction. Only a few students are engaged, with one or two students demonstrating some disturbing behaviour: two students, S09 and S15, occasionally disturbed the lesson, students S01 and S13 were off-task helping students S10 and S11. There appear to be some 'sub-groups' i.e. clusters of students who interact more often with each other. The second lesson (R4) is a year 7 maths lesson the area of a triangle. The lesson starts with a short instruction, followed with individual work with the teacher walking around. There is a lot of individual interaction between teacher and students. Towards the end of the lesson the teacher uses software to quiz the students. As could be seen in figure 2c, the Lesson Note app provides basic statistics for the 
lessons as well. These statistics already convey that lesson R1 was much more 'whole class' than R4. After exporting the data and applying the SNA methodology, table 3 shows the resulting whole-lesson networks and some appropriate metrics. It further confirms that for R1 the in-degree of E, the entire class, is highest but that teacher talk was relatively limited.

Table 3. Networks and some basic metrics for two of the five maths lessons.

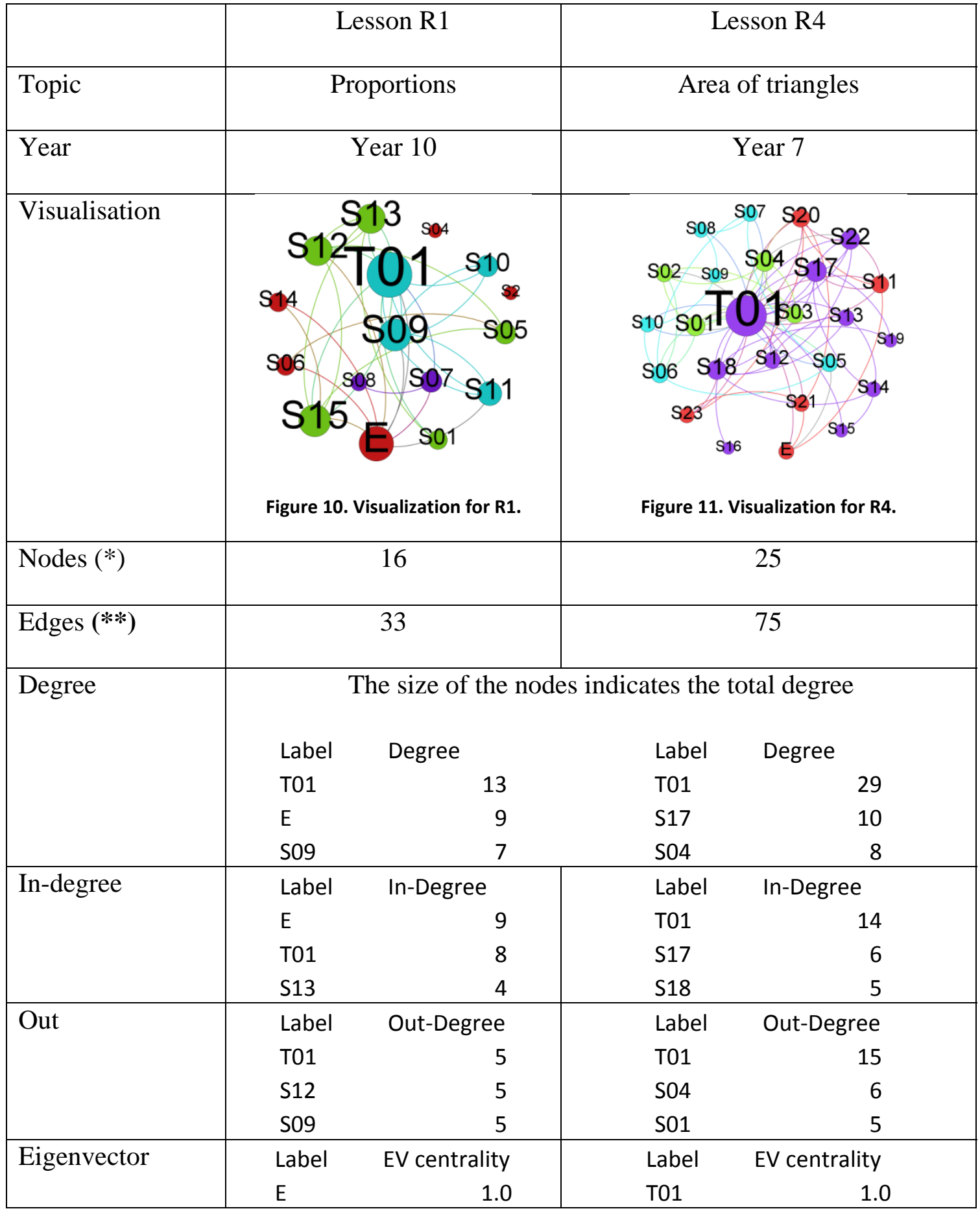




\begin{tabular}{|c|c|c|c|c|}
\hline \multirow[t]{4}{*}{ centrality } & T01 & 0.85 & S17 & 0.60 \\
\hline & S13 & 0.60 & S18 & 0.55 \\
\hline & S09 & 0.49 & S20 & 0.50 \\
\hline & S15 & 0.43 & S23 & 0.48 \\
\hline Average degree & \multicolumn{2}{|c|}{2.062} & \multicolumn{2}{|c|}{3.0} \\
\hline Avg clust.coeff. & \multicolumn{2}{|c|}{0.334} & \multicolumn{2}{|c|}{0.322} \\
\hline Modularity & \multicolumn{4}{|c|}{ The colours of the nodes denote to which sub-group nodes belong. } \\
\hline
\end{tabular}

(*) Nodes might include a node E for 'Entire class'. The originally included D for 'devices' used has been omitted.

$(* *)$ Note that the number of edges does not reflect the total number of notes taken for two reasons. One

is the removal of some notes as described in the data processing section. Another reason is the aggregation of edges between two nodes into one edge with dynamic timestamps.

The basic picture shows one lesson, R4, on average having more interactions. As explained previously, apart from looking at the whole network it also is possible to look at measures for individual nodes, like degree and centrality. For example, in both R1 and R4 the total degree for the teacher T01 is highest, indicating a prominent role for the teacher. However, in R1 the student S12 has the same out-degree as the teacher. R1 also shows two nodes with an in-degree of 0, namely S02 and S04: the students were not spoken to during the lesson, nor interacted. Of course, they were part of E, the entire class. There were no solitary nodes in R4. This might be seen as useful information with regard to classroom dynamics. When it comes to the largest Eigenvector centrality measure behind the teacher and 'whole classroom', S13, S09 and S15 show the highest values in R1, which confirms their central role in the classroom process. The same can be said for S17, S18, S20 and S23 in R4: they have high values (behind the teacher in this case) of Eigenvector centrality. It is suggested that these measures might indicate key actors in the classroom: knowing these might be helpful in addressing (negative or positive) behaviour. Of course there also are 'lowest values' for each of these metrics. 
The modularity metric shows that 'sub groups' exist. Although the particular layout chosen does not convey the spatial classroom aspect, it is evident that closely seated students interact more with each other than separated students. This in itself might be less interesting than 'exceptions to the rule', namely students that interact 'outside' their immediate peers. A final aspect that can be looked at is the dynamic, temporal aspect: how does classroom interaction change over time?

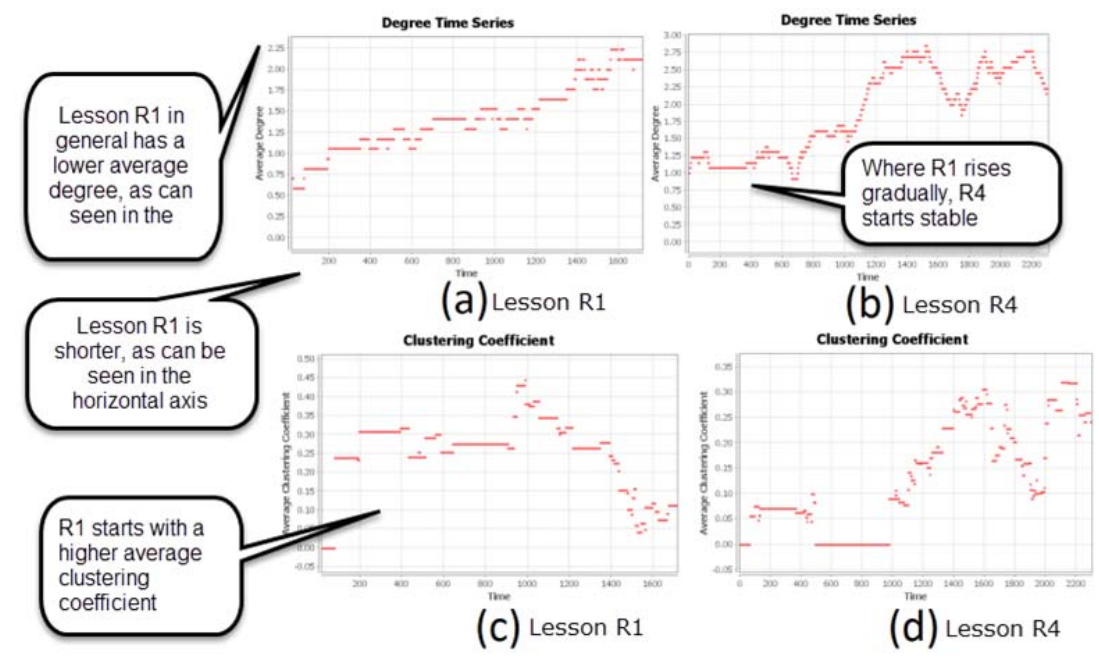

Figure 12. Graphs for lessons R1 and R4 for average degree (a and b) and average clustering coefficient, respectively (c and d).

$\left(^{*}\right)$ Note that graphs presented can only present the temporal aspect by choosing a 'window size'. If the window would span from the beginning to the end of the lesson the 'whole network' measures would be obtained. With a smaller window the measure would be calculated for that one window, increased by a designated value (pitch) and calculated again. In these examples a window size of 500 and pitch of 1.0 were chosen. For more information, see Moody, McFarland and Bender-deMoll (2005).

Figure 12 shows that during the lessons R1 and R4 the average degree and clustering coefficient follow different patterns. The interpretation of these dynamic aspects can be difficult. In general it shows, by observing the vertical axis, that R4 consistently has a higher average degree and thus interaction during the lesson. R4 also is the longer of the two lessons. However, whereas R1 increases steadily, R4 first is quite stable. This seems consistent with the teacher first doing instruction and then letting students work individually (and not enforcing total silence). The dip at the end of R4 might be the 
teacher taking the lead again, with an interactive end-of-lesson-quiz. When it comes to clustering it seemed that R1 had more distinctive 'cliques' or 'sub-groups' from the beginning of the lesson than R4. This seems to correspond with the apparent disturbances in R1. By adding together all the time intervals for a particular edge, for example those edges that are directed to the teacher T01, it also is possible to determine the amount of 'help seeking' students do. Metrics like these can subsequently be included in more advanced longitudinal statistical models, in an attempt to discover 'patterns of teaching'. This is not straightforward and some thoughts on the challenges are presented at the end of the paper.

\section{Conclusions and discussion}

This article set out to demonstrate three things. Firstly, to explore how technology can be used to capture classroom interaction data. The development of apps like Lesson Note that facilitate observational methods can be described as a useful application of technology for capturing classroom data. These methods complement technical developments for video since the TIMSS 1999 study. Although recording classrooms technically has become much easier, ethical and privacy challenges might make the use of observation apps more useful. In the article I have tried to show that methodologically both indirect and direct observational methods can be used with SNA techniques. The examples provided demonstrate that both video and app data can be used as source data for classroom observations. Secondly, the advent of social network analyses methods, including the dynamic version of it, can provide valuable insight in classroom dynamics between actors. The visualizations described and accompanying measures convey patterns of interaction and may highlight features of individual nodes and sub-groups. Thirdly, the complex longitudinal data that dynamic social network analysis generates can be visualized and modelled with technology. By describing 
classroom observations in a visual and quantified way it might be possible to use advanced statistical methods to indicate overall patterns. Of course, as Howe and Abedin (2013) indicate, whether quantitative methods provide a useful proxy or oversimplify classroom practice, is a risk but if the methodology proves useful it would provide a magnitude of any effects. All this demonstrates, in my view, that there are opportunities in using technology and social network analysis in research, both at data collection and data analysis level. It is suggested that the potential of these developments should be explored further and utilized for more lessons and more different lessons, as it is also clear there is still a way to go when it comes to fully embrace the methodology. I would like to conclude with some discussion on three aspects.

A first aspect concerns, as always with statistical data, interpretation: what exactly do the visualizations and metrics say? How do we interpret these? Not all metrics from SNA might be just as informative. It would be all too easy to just look at the numbers and draw some quick conclusions. This aspect touches on the decades-old discussion touched upon in the first part of the article: would a quantitative approach do justice to the rich context of a classroom? The difficulty here is that what critics identify as weaknesses of 'field coding' methods its practitioners regarded as being among its strengths; a deliberately detached outsider's view, the insistence on knowing what to look for through the use of protocols, and the "consequent restricted focusing on what lay within the observers frame of reference” (Edwards and Westgate 1994, 87). On its own it is put forward that SNA's quantitative features, like sociometrics, already can serve as a "reasonable proxy for the more nuanced picture that qualitative approaches reveal” (Howe and Abedin 2013, 333). In addition to this SNA can also include qualitative data, demonstrated partly by the notes taken with the Lesson Note app. 
Essentially, together with triangulation of data, complementing visualizations and metrics with other data sources through a mixed methods approach SNA can be a welcome addition to the research methods repertoire. This emphasis corresponds with the mixed methods approach the Economic and Social Research Council in the UK conveyed in one of its reports (Edwards 2010). In my view this also means that more interdisciplinary work needs to take place, whereby educational researchers, methodologists and educators collaborate. It is hoped that with appropriate technologies and methodologies the education research field will take on the challenge described in the introduction (Howe and Abedin 2013) and cautiously might describe patterns of classroom interaction.

A second aspect concerns the quality of the data. As the examples in the analyses have shown the data that is captured needs to be of sufficient quality. This means that data at least needs to have information about senders, receivers and temporal data. The information can be augmented by attributes for nodes and edges; these attributes can be relatively simple like gender or achievement, but can also be qualitative data like text documents. However, these analyses show that even with these methodological advances there are plenty of challenges in a simple temporal network, let alone if one wants to add extra attributes to the network(s). Not only is this a very big challenge for one project, if we would like to compare lessons it becomes even more imperative that clear protocols are used for gathering observation data or recording lessons. One example to demonstrate this lies in the way one records whole-class interactions: does one model this as interaction to every individual node or to one node that represents the 'whole classroom'. The decision by an observer to qualify an interaction as 'whole class' needs to be uniform within- and between-classes. The same question is relevant for groups within classes. Whatever the answer one consistent 
protocol will at least guarantee that results remains comparable and might be generalized, fully realizing that closed protocols lie on a tension with the first aspect. Perhaps the observation process can also be improved by promoting improvements to software, for example like the recommendations for the Lesson Note app as described by Bokhove (2015).

A third and final aspect that might become more and more important during data collection concerns finding study participants. During this project schools seemed to be more and more hesitant about participating in a project that involved videoing classrooms. It was the main cause that data collection ended relatively late in the study. There seem to be two main reasons for this. One is anxiety with recording, especially in relationship to (ethical) approval from senior leadership, students and parents. With the advent of mobile devices it seems that stakeholder are increasingly cautious in giving consent for filming. For existing data, secondary data, an important issue concerns whether the original study participants were aware of future uses of the data. It might be tempting to assume that research on 'normal classroom practice' could be collected, but researchers will need to take into account appropriate ethical measures (Wiles, Clark, and Prosser 2011). Another reason concerns the logistics of installing cameras, staffing and arranging resources. One affordance of using observation apps seems to be the fact that both of these objections from schools are moderated: apps are seen as less intrusive and quickly set up. It would also be good to get more out of existing secondary data sets, including videos, that are already out there. One such, albeit dated, example is the TIMSS video study. There, however, are more recent examples as well. It could prevent everyone reinventing the wheel and could consolidate valuable resources. It is a key reason why this article wanted to address the application of SNA for both indirect and direct observational methods. 
This article has set out to describe the application of dynamic Social Network Analysis techniques to classroom interaction. It tried to address the challenge of reviving a quantitative approach (but not excluding qualitative aspects) with a temporal aspect. It is my belief that the proposed method at the least provides a relevant 'lens' in any mixed methods approach towards classroom interaction, with potentially more scope for discovering classroom interaction patterns. Revisiting the initial aims, the take away points for this methodology and recommendations are:

1. SNA provides a standardised method to compare a wide range of classroom interaction contexts like classroom videos and different data types from observations. It is expected that he standardized nature of the proposed methodology allows for easier generalization through inclusion of contextual factors (cultural, structural and population differences), for example through the construction of 'lesson signatures'.

2. It can use this same standardised methodology to add a temporal layer which can be used to study classroom interactions over time: dynamic Social Network Analysis.

3. The methodology provides the opportunity to use both quantitative analysis and qualitative analysis within one framework.

4. It adds an instantly appealing visual aspect to classroom interaction, both at a cross-sectional level as the development over time. The principles can usefully be supported by a variety of software, ranging from more visually oriented software like Gephi, and software for statistical modelling (e.g. changes in social networks over time).

5. Further exploration of the potential of new technology, like in using tablet-based applications to collect classroom interaction data, both longitudinal and cross- 
sectional, would be useful. An emphasis on network features might provide an important focus of study.

Needless to say, this can only be found out by exploring this route further, this article hopes to provide an important step.

\section{Acknowledgements}

I would like to thank Thomas McDougal from the Lesson Study Alliance for kindly providing some data from junior high schools in the USA captured by their Lesson Note app to study the workings of the app. I would also like to thank Dr. Hazel Brown for some data processing. Also thanks to staff and students of the participating school. Finally, a big thanks to all the reviewers. This project was funded by the Strategic Interdisciplinary Research Development Fund of the University of Southampton. Ethics approval was granted by the university, under number 9898 .

\section{References}

Bokhove, C. 2015. “Lesson Note app.” International Journal of Research \& Method in Education 38 (3): 338-339. doi:10.1080/1743727X.2015.1030134

Baker, R.S, A.Salvi, M. van Velsen, and A. Whiting. HART -- Human Affect Recording Tool. Android software. U.S. Army Research Laboratory, 2013.

Banks, D., and K.M. Carley. 1996. ”Models of Social Network Evolution.” Journal of Mathematical Sociology 21 (1-2): 173-196.

Barabasi, A-L. 2002. Linked: The New Science of Networks. Cambridge, MA: Perseus Publishing.

Bender-deMoll, S. ndtv: Network Dynamic Temporal Visualizations (version 0.5.1). R package software. 2014.

Bender-deMoll, S., and D.A. McFarland. 2006. "The Art and Science of Dynamic Network Visualization.” Journal of Social Structure 7 (2).

Choudhury, T., and A. Pentland. 2004. "Characterizing social networks using the sociometer.” In Proceedings of CASOS 2004 NAACSOS Conference.

Coburn, C.E., and J.L. Russell. 2008. “District Policy and Teachers’ Social Networks.” Educational Evaluation and Policy Analysis 30 (2): 203-235. 
Cohen, L., L. Manion, and K. Morrison. 2011. Research Methods in Education. 7th ed. London: Routledge Falmer.

Creemers, B. P. M., and L. Kyriakides. 2008. The Dynamics of Educational Effectiveness: A Contribution to Policy, Practice and Theory in Contemporary Schools. London: Routledge.

Daly, A. J., and K.S. Finnigan. 2010. “A Bridge Between Worlds: Understanding Network Structure to Understand Change Strategy.” Journal of Educational Change 11 (2): 111-138.

Doreian, P., and F. Stokman. 1997. "The Dynamics and Evolution of Social Networks.” In Evolution of social networks, edited by P. Doreian and F. Stokman, 1-17. Abingdon: Routledge.

Dunne, J., R. Williams, and N. Martinez. 2002. “Food-web Structure and Network Theory: The Role of Connectance and Size.” Proceedings of the National Academy of Science 99 (20): 12917-12922. doi:10.1073/pnas.192407699

Edwards, G. 2010. Mixed-method Approaches to Social Network Analysis. ESRC National Centre for Research Methods Review paper.

Edwards, A.D., and D.P.G. Westgate. 1994. Investigating Classroom Talk. 2nd ed. London: The Falmer Press.

Freeman, L. 2004. The Development of Social Network Analysis: A Study in the Sociology of Science. Vancouver, BC, Canada: Empirical Press.

Fruchterman, T. M. J., and E.M. Reingold. 1991. “Graph Drawing by Force-Directed Placement.” Software: Practice and Experience 21 (11): 1129-1164. doi:10.1002/spe.4380211102

Gephi Consortium. Gephi (version 0.8.2 beta). Windows software. 2013.

Givvin, K.B., J. Hiebert, J. Jacobs, H. Hollingsworth, and R. Gallimore. 2005. “Are There National Patterns of Teaching? Evidence from the TIMSS 1999 Video Study." Comparative Education Review 49 (3): 311-343.

Hansberger, J. Work Observer. Apple iOS software. 2011.

Handcock, M.S., D. Hunter, C. Butts, S. Goodreau, P. Krivitsky, S. Bender-deMoll, and M. Morris. Statnet: Software Tools for the Statistical Analysis of Network Data (version 2014.2.0). R package software. 2014.

Handcock, M.S, D. Hunter, C. Butts, S. Goodreau, and M. Morris. 2008. "Statnet: Software Tools for the Representation, Visualization, Analysis and Simulation of Network Data.” Journal of Statistical Software 24 (1): 1-11. 
Hattie, J. 2009. Visible Learning: A Synthesis of over 800 Meta-analyses Related to Achievement. London: Routledge.

Hiebert, J., R. Gallimore, H. Garnier, K.B. Givvin., H. Hollingsworth, J. Jacobs, A.M. Chui et al. 2003. Teaching Mathematics in Seven Countries: Results from the TIMSS 1999 Video Study. U.S. Department of Education. Washington, DC: National Center for Education Statistics.

Howe, C., and M. Abedin. 2013. “Classroom Dialogue: A Systematic Review Across Four Decades of Research.” Cambridge Journal of Education 43 (3): 325-356. doi:10.1080/0305764X.2013.786024

Jacobs, J.K., H. Hollingsworth, and K.B. Givvin. 2007. “Video-based Research Made "Easy”: Methodological Lessons Learned from the TIMSS Video Studies." Field Methods 19 (3): 284-299. doi:10.1177/1525822X07302106

Janík, T., and T. Seidel, eds. 2009. The Power of Video Studies in Investigating Teaching and Learning in the Classroom. 1st ed. Münster: Waxmann. Jin, E.M., M. Girvan, and M.E.J. Newman. 2001. “The Structure of Growing Social Networks.” Phys. Rev. E 64: 046132. doi:10.1103/PhysRevE.64.046132

Krivitsky, P.N., and M.S. Handcock. 2014. “A Separable Model for Dynamic Networks.” Journal of the Royal Statistical Society: Series B (Statistical Methodology) 76 (1): 29-46. doi:10.1111/rssb.12014

McCulloh, I., H.L. Armstrong, and A.N. Johnson. 2013. Social network analysis with applications. Hoboken, New Jersey: John Wiley \& Sons, Inc.

Lesson Study Alliance. Lesson Note. Apple iOS software. 2014.

Li, L., Z. Shouhui, and C. Xinying. 2011. "Beyond Research: Classroom Interaction Analysis Techniques for Classroom Teachers.” Paper presented at the Redesigning Pedagogy International Conference, Singapore, May 30-June 1.

Moody, J., D.A. McFarland, and S. Bender-deMoll. 2005. “Dynamic Network Visualization: Methods for Meaning with Longitudinal Network Movies.” American Journal of Sociology 110 (4): 1206-1241.

Muijs, R.D., and D. Reynolds. 2001. "Student Background and Teacher Effect on Achievement and Attainment in Mathematics: A Longitudinal Study.” Paper presented at the International Congress for School Effectiveness and Improvement, Toronto, January 5-9.

Muijs, R.D., and D. Reynolds. 2010. Effective Teaching: Research and Practice. London: Paul Chapman. 
Penuel, W., M. Riel, A. Krause, and K. Frank. 2009. “Analyzing Teachers’ Professional Interactions in a School as Social Capital: A Social Network Approach.” The Teachers College Record 111 (1): 124-163.

Pitts, V.M., and J. Spillane. 2009. “Using Social Network Methods to Study School

Leadership.” International Journal of Research and Method in Education 32 (2): 185-207.

Prell, C. 2011. Social Network Analysis. London: SAGE.

Ripley, R., K. Boitmanis, and T.A.B. Snijders. RSiena: Siena - Simulation Investigation for Empirical Network Analysis (version 1.1-232). R package software. 2013.

Robins, G., P. Pattison, Y. Kalish, and D. Lusher. 2007. “An Introduction to Exponential Random Graph (p) Models for Social Networks.” Social Networks 29 (2): 173-191.

Scheerens, J., and R. Bosker. 1997. The Foundations of School Effectiveness. Oxford: Pergamon Press.

Scott, J. 2013. Social Network Analysis. London: SAGE.

Scott, P. and J. Ametller. 2007. “Teaching Science in a Meaningful Way: Striking a Balance Between ‘Opening up’ and ‘Closing down’ Classroom Talk.” School Science Review 88 (324): 77-83.

Scott, P.H., Mortimer, E.F. and O.G. Aguiar. 2006. “The Tension Between Authoritative and Dialogic Discourse: A Fundamental Characteristic of Meaning Making Interaction in High School Science Lessons.” Science Education 90 (4): 605-631.

Sentse, M., N. Kiuru, N., R. Veenstra, and C. Salmivalli. 2014. “A Social Network Approach to the Interplay Between Adolescents' Bullying and Likeability Over Time.” Journal of Youth and Adolescence 43 (9): 1409-1420.

Snijders, T.A.B. 1996. “Stochastic Actor-oriented Models for Network Change.” Journal of Mathematical Sociology 21 (1-2): 149-172.

Snijders, T.A.B. 2001. “The Statistical Evaluation of Social Network Dynamics.” Sociological Methodology 31 (1): 361-395. doi:10.1111/0081-1750.00099

Teddlie, C., and D. Reynolds. 2000. The International Handbook of School Effectiveness research. London: Falmer Press. 
Wasserman, S., and P. Pattison. 1996. "Logit Models and Logistic Regressions for Social Networks: I. An Introduction to Markov Graphs and P*.” Psychometrika 61 (3): 401-425.

Wiles, R., A. Clark, and J. Prosser. 2011. "Visual Research Ethics at the Crossroads.” In The SAGE Handbook of Visual Research Methods, edited by E. Margolis and L. Pauwels, 685-706. Thousand Oaks, CA: SAGE Publications. 


\begin{tabular}{|c|c|c|c|}
\hline & Lesson R2 & Lesson R3 & Lesson R5 \\
\hline Topic & Polynomials & Vectors, statistics & Standard form \\
\hline Year & Year 12 & Year 13 & Year 9 \\
\hline Visualisation & 808 & $\begin{array}{c}\mathrm{s} 01 \quad \mathrm{~S} 08 \\
\mathrm{~s} 02 \mathrm{SO} 05 \mathrm{~S} 04 \\
\mathrm{~S} 09 \mathrm{TO} \\
\mathrm{s} 03 \mathrm{~S} 07 \mathrm{~S} 10\end{array}$ & 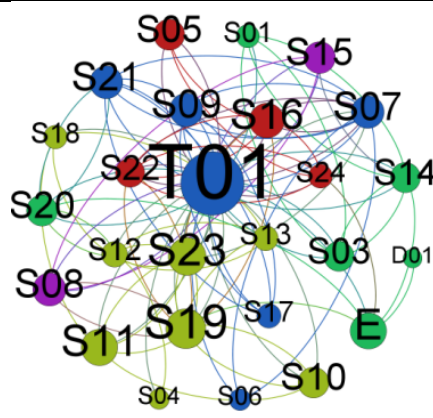 \\
\hline Nodes $(*)$ & 13 & 12 & 26 \\
\hline Edges $(* *)$ & 38 & 39 & 102 \\
\hline Degree & \multicolumn{3}{|c|}{ The size of the nodes indicates the total degree } \\
\hline Average degree & 2.923 & 3.25 & 3.923 \\
\hline Avg clust.coeff. & 0.399 & 0.305 & 0.496 \\
\hline Modularity & \multicolumn{3}{|c|}{ The colours of the nodes denote to which sub-group nodes belong. } \\
\hline
\end{tabular}


Supplemental material. Networks and some basic metrics for three additional maths lessons.

$(*)$ Nodes might include a node $\mathrm{E}$ for 'Entire class' and D for an attribute used in class like a projector.

$(* *)$ Note that the number of edges does not reflect the total number of notes taken for two reasons. One is the removal of some notes as described in the data processing section. Another reason is the aggregation of edges between two nodes into one edge with dynamic timestamps added. 